\title{
Reflets
}

Revue ontaroise d'intervention sociale et communautaire

\section{Solidarité et citoyenneté : initiatives pour contrer la pauvreté chez les francophones dans Ottawa-Carleton}

\section{Cécile Coderre et Madeleine Dubois}

Volume 6, numéro 2, automne 2000

Problèmes sociaux en Ontario français

URI : https://id.erudit.org/iderudit/026316ar

DOI : https://doi.org/10.7202/026316ar

Aller au sommaire du numéro

Éditeur(s)

Reflets : Revue ontaroise d'intervention sociale et communautaire

ISSN

1203-4576 (imprimé)

1712-8498 (numérique)

Découvrir la revue

Citer cet article

Coderre, C. \& Dubois, M. (2000). Solidarité et citoyenneté : initiatives pour contrer la pauvreté chez les francophones dans Ottawa-Carleton. Reflets, 6(2),

61-86. https://doi.org/10.7202/026316ar

Tous droits réservés (C) Reflets : Revue ontaroise d'intervention sociale et communautaire, 2000
Ce document est protégé par la loi sur le droit d'auteur. L'utilisation des services d’Érudit (y compris la reproduction) est assujettie à sa politique d'utilisation que vous pouvez consulter en ligne.

https://apropos.erudit.org/fr/usagers/politique-dutilisation/ 


\section{Solidarité et citoyenneté $\square$ :}

\section{initiatives pour contrer la pauvreté chez les francophones dans Ottawa-Carleton}

"...la pauvreté et les personnes qui en sont marquées demeurent néanmoins une problématique fréquemment occultée dans l'histoire de la communauté francoontarienne."
Cécile Coderre et Madeleine Dubois ${ }^{1}$

École de service social de l'Université d'Ottawa

Sur tous les murs de nos villages

dans notre langue couleur terre couleur misère

Nous avons égratigné à même les ongles de nos mains sales de travailleurs, les lettres et les visages de notre Histoire.

Jean-Marc Dalpé 1980

Même si quelques-unes des oeuvres marquantes de la poésie franco-ontarienne se veulent «avant tout, une défense des plus petits, des défavorisés, des déracinés » (Paré 1994 :128), la pauvreté et les personnes qui en sont marquées demeurent néanmoins une problématique fréquemment occultée dans l'histoire de la communauté franco-ontarienne. Les luttes dont il est question dans son historiographie et dans sa littérature s'articulent surtout autour des thèmes de l'éducation, même si elles se sont élargies dans les vingt dernières années aux champs de la culture et des droits juridiques (Juteau et Séguin-Kimpton 1993; Carrière 1993). La défense des droits des plus pauvres ne fait pas partie des 
"...hormis quelques brèches émanant de prises de conscience attribuables au mouvement des femmes et à certaines pratiques d'alphabétisation, un voile de silence a continué d'entourer, jusqu'à tout récemment, les pauvres de la communauté francoontarienne... » revendications traditionnelles des associations franco-ontariennes ni des préoccupations exprimées par leurs dirigeants (Welch 1995). Comme le note Brigitte Bureau dans son analyse de vingt ans de luttes menées par l'Association canadienne-française de l'Ontario (ACFO), celle-ci n'aborde pas la question de la pauvreté. Tout se passe comme si, après avoir été victime d'une discrimination historique dans le marché du travail (Farmer et Poirier 1999: 275), le rattrapage que l'on connaît aujourd'hui gommait les inégalités qui perdurent au sein de la communauté francoontarienne ${ }^{2}$. Somme toute, hormis quelques brèches émanant de prises de conscience attribuables au mouvement des femmes et à certaines pratiques d'alphabétisation, un voile de silence a continué d'entourer,jusqu'à tout récemment, les pauvres de la communauté franco-ontarienne, qui semblent n'exister ni comme catégorie, ni comme groupe, ni même comme citoyens.

Le présent article s'inscrit dans un corpus naissant d'écrits publiés au cours des dernières années (Diallo et Reitsma-Street 1995; Mongrain 1997; Welch 1995;1999) qui traitent de questions sociales liées, notamment, à la question de la pauvreté dans le milieu franco-ontarien. Notre but est de documenter les initiatives contre la pauvreté ayant vu le jour au cours de la dernière décennie au sein d'organismes communautaires offrant des services à la population francophone d'Ottawa-Carleton et d'analyser la nature ainsi que la portée de certaines de ces pratiques. Afin de mieux saisir le contexte les ayant engendrées, nous présenterons d'abord succinctement certains rapports et documents récents ayant trait à la situation sociale et économique des Franco-Ontariennes et des Franco-Ontariens, pour ensuite brosser un portrait des enjeux et des paradoxes plus spécifiques liés à la problématique de la pauvreté chez les francophones d'Ottawa-Carleton. Les limites du présent article ne permettant pas une analyse de l'ensemble des initiatives contre la pauvreté, nous avons choisi de cibler plus particulièrement celles concernant la sécurité alimentaire, tout en étant conscientes de la nécessité d'adresser, dans un article subséquent, les problèmes, tout aussi importants pour les personnes vivant dans la précarité, de l'emploi, du logement et des enjeux qui y sont liés. 
Comme les pratiques communautaires en Ontario français sont peu documentées, la recherche sur laquelle s'appuie cet article revêt forcément un caractère exploratoire. Elle se fonde, dans un premier temps, sur une lecture de divers rapports gouvernementaux et sur des rapports d'organismes qui apportent un certain éclairage sur la communauté franco-ontarienne, eu égard, notamment, à certaines particularités de la pauvreté qu'on y vit. L'étude s'appuie également sur le dépouillement de diverses archives d'organismes communautaires d'Ottawa-Carleton, notamment Entraide budgétaire et les Centres communautaires francophones de la région ${ }^{3}$, ainsi que sur l'analyse du contenu des rapports annuels de ces derniers et ce pour les dix dernières années.

\section{Pratiques communautaires et pauvreté $\square$ : quelques brèches dans une histoire marquée par l'invisibilité}

"...ces comités de citoyens francophones s'élargissent à de nouveaux champs d'intervention, qui marquent une rupture avec les pratiques traditionnelles, en se situant " hors de la seule logique de l'Église catholique et des écoles »."
Le Comité du Réveil de la Basse-Ville d'Ottawa, créé au cours des années 60 pour donner une voix à la population majoritairement francophone de la Basse-Ville, confrontée à la démolition de son quartier dans le contexte d'un projet de rénovation urbaine, offre un des premiers exemples de mobilisation communautaire ponctuelle (Fonds d'archives du Comité de Réveil). Après avoir participé avec une trentaine de groupes de quartiers de la ville d'Ottawa pour s'opposer au vaste projet de rénovation urbaine, le Comité de Réveil de la Basse-Ville sera dissous à la fin des années 70. Comme d'autres créés à l'époque, ce comité appartient au mouvement communautaire plus large que Bagaoui et Dennie associent au « type de syndicalisme du cadre de vie » (1999 : 80). Tout en exerçant des pressions auprès des gouvernements pour réclamer des équipements et des services collectifs pour la population de leur quartier, ces comités de citoyens francophones s'élargissent à de nouveaux champs d'intervention, qui marquent une rupture avec les pratiques traditionnelles, en se situant « hors de la seule logique de l'Église catholique et des écoles ». 
En effet, par leurs interventions dans l'amélioration des conditions de vie des citoyens et des services collectifs : services de transport, de médias francophones, d'entretien domiciliaire, de services pour les aînés, des réseaux d'onde, etc, ces comités tentent d'arracher les associations du paternalisme des élites et de l'encadrement paroissial (Bagaoui et Dennie 1999 : 81).

Au début des années 80, le mouvement d'alphabétisation populaire (Wagner 1991) et la prise en considération par l'ACFO de la réalité des Franco-Ontariens et des Franco-Ontariennes analphabètes ou sous-scolarisés ouvrent une autre brèche importante dans le silence entourant les Sans voix (ACFO 1985). En effet, les données sur l'analphabétisme des francophones en Ontario sont désastreuses au début des années $80: 6,1 \%$ de la population serait complètement analphabète, alors qu'un autre $22,5 \%$ le serait fonctionnellement, comparativement à 14,1\% des Ontariens non francophones. C'est l'ensemble du système social qui est mis en cause dans l'explication de cette situation qui interpelle toute la communauté franco-ontarienne :

Conditions de vie difficiles, activités économiques faisant obstacle à l'alphabétisation, enseignement en anglais imposé, expérience négative de l'école pour les francophones de milieux populaires et survie du français assurée par les traditions orales (Institut canadien d'éducation des adultes 1989 : 85).

"À la fin des années 80 , plusieurs projets d'alphabétisation privilégient des pratiques qui s'inscrivent dans une approche conscientisante. "
Le réseau qui se crée autour de projets d'alphabétisation donne lieu à l'établissement d'une concertation régionale et provinciale. Ce réseau touche une population marginalisée, exclue souvent des associations et des institutions culturelles franco-ontariennes. Les centres d'alphabétisation deviennent en fait des lieux symboliques importants dans le mouvement de démocratisation des institutions franco-ontariennes (Gilbert 1999 : 69) ${ }^{4}$. À la fin des années 80, plusieurs projets d'alphabétisation privilégient des pratiques qui s'inscrivent dans une approche conscientisante. Celles-ci marquent un certain renouveau, voire même une 
"...ces diverses activités ont certainement permis une réflexion sur les structures de marginalisation sociale des francophones, réflexion qui, quelques années plus tard, verra l'émergence de pratiques favorisant la participation citoyenne... des personnes souvent laissées pour compte, ..." transformation de certaines pratiques communautaires ponctuelles amorcées au cours des années 60 .

Le début des années 80 est aussi associé à la transformation de regroupements de femmes ontaroises particulièrement préoccupées par l'inégalité sociale et économique des femmes (Cardinal 1998; Cardinal et Coderre 1990) et par le même fait, par la précarité économique de ces dernières. Ainsi, en 1983, la Fédération des femmes canadiennes-françaises (FFCF) publie l'étude Seules en grand nombre, Dossier socio-économique sur la situation des Ontaroises chefs de famille monoparentale. Elle dénonce la situation de $59 \%$ des familles monoparentales vivant en dessous du seuil de la pauvreté dans la région d'Ottawa-Carleton. Par ailleurs, le Centre d'accès pour femmes (Ottawa) soutenait déjà les femmes dans leur démarche d'autonomie financière et quelques groupes de femmes chefs de famille monoparentale s'organisaient aussi dans le Nord et l'Est ontarien (FNCFC 1986 :21).

Ces efforts pour rejoindre une population francophone marginalisée constituent en quelque sorte un terreau sur lequel pourront s'ancrer les pratiques communautaires des années 90 . Il faut ajouter à cette conjoncture favorable, le pouvoir symbolique de la Loi 8 (Andrew et al: $1997: 15$ ) de même que le développement de nouvelles pratiques socio-économiques telles les coopératives de logement et les garderies à but non lucratif (Welch 1999 : 62). Dans leur ensemble, ces diverses activités ont certainement permis une réflexion sur les structures de marginalisation sociale des francophones, réflexion qui, quelques années plus tard, verra l'émergence de pratiques favorisant la participation citoyenne (Lamoureux 2000) des personnes souvent laissées pour compte, pratiques qui par ailleurs facilitent l'« émancipation collective» (Brun 1996 : 131) de la communauté.

\section{Bref profil de la pauvreté dans Ottawa-Carleton}

Parler de la pauvreté dans une région comme Ottawa-Carleton, qui connaît une croissance économique inégalée, peut sembler 
«... l'écart entre les ménages à revenu élevé et ceux à faible revenu s'accroît également : "la polarisation des revenus est plus prononcée dans la municipalité d'Ottawa-Carleton et à Ottawa qu'en Ontario et au Canada "..." " paradoxal. En effet, deux indices, soit le taux de chômage et le revenu moyen familial, offrent l'image d'une région prospère. Ainsi le taux de chômage demeure sous les moyennes nationale et provinciale (Conseil de planification sociale d'Ottawa-Carleton (CPSOC) 1999a : 7). De plus, "le revenu familial moyen s'établissait en 1995 à 67871 \$ comparativement à 55247 \$à l'échelle nationale"(CPSOC 1999a : 4). Mais paradoxe il y a. En effet, l'écart entre les ménages à revenu élevé et ceux à faible revenu s'accroît également : "la polarisation des revenus est plus prononcée dans la municipalité d'Ottawa-Carleton et à Ottawa qu'en Ontario et au Canada " (CPSOC 1999b : 14). De plus, même si le revenu moyen des familles a connu une croissance au niveau de la municipalité régionale, celui des familles et des ménages dans la ville d'Ottawa a diminué entre 1985 et 1995, ce qui confirme la polarisation croissante des revenus entre le noyau urbain et les zones périphériques. Enfin, à Ottawa-Carleton, la proportion globale de personnes et de familles à faible revenu s'accroît de façon constante (CPSOC 1999a : 77). C'est ce qui inspire le titre de la publication du Conseil de planification sociale d'Ottawa-Carleton et de Centraide d'Ottawa-Carleton, Une histoire de deux mondes (1999).

Plusieurs groupes sont particulièrement vulnérables : les jeunes, les familles monoparentales et les nouveaux arrivants. En particulier "chez les personnes de moins de 35 ans, le taux de pauvreté ${ }^{5}$ se maintient au dessus de la moyenne nationale " (CPSOC 1999b :14). Le taux de chômage chez les personnes de 20 à 24 ans a quant à lui grimpé de 9,4\% à 13,7 \% entre 1987 et 1997 (CPSOC 1999a : 4).

La situation pour les familles démunies est difficile mais elle l'est encore plus pour les familles gynéparentales ${ }^{6}$. Ces familles sont concentrées àVanier (28,1\%) et à Ottawa (19,7\%) (CPSOC : 1999a). On estime que la proportion de faible revenu pour les familles monoparentales est de $41,8 \%$ et $37,6 \%$ à Vanier et à Ottawa respectivement (CPSOC 1999 a : 80). C'est ce qui fait dire au CPSOC que

...ces femmes sont extrêmement vulnérables à une perte de leur revenu, ont moins de latitude sur le marché $d u$ 
travail et ont besoin d'une aide particulière pour être en mesure de travailler, notamment des services de garde d'enfants subventionnés et un soutien domestique (CPSOC 1999a: 26).

"Une autre population vulnérable est constituée par les nouvelles arrivantes et les nouveaux arrivants de même que par les personnes de minorités visibles. "

"Cette discrimination dans le marché $d u$ travail a aussi une composante francophone et elle se manifeste dans l'ensemble de la province."
Une autre population vulnérable est constituée par les nouvelles arrivantes et les nouveaux arrivants de même que par les personnes de minorités visibles. La région d'Ottawa-Carleton a connu, entre 1986 et 1993, une forte croissance de la population d' immigrants avec un nombre important de réfugiées et de réfugiés. Certaines de ces personnes, confrontées notamment à des barrières linguistiques et à la non reconnaissance de leurs compétences, rencontrent des difficultés dans la recherche d'emploi. Ainsi en 1991,10 \% de la population immigrante d'Ottawa-Carleton était composée de professionnels sous-employés (Huntley 1998). Cette discrimination dans le marché du travail a aussi une composante francophone et elle se manifeste dans l'ensemble de la province. Parmi les francophones membres d'une minorité raciale en Ontario, le taux de chômage chez les hommes de 15 à 64 ans atteint 14,8\% comparativement à $12,2 \%$ chez les nonfrancophones de minorité raciale et à $8,7 \%$ chez les nonfrancophones (Farmer et Poirier 1999 : 275).

Au sein de la population francophone, qui représente $17 \%$ de la région d'Ottawa-Carleton, la réalité de la pauvreté est certes difficile à mesurer, mais certains indices nous permettent de saisir qu'elle est bien réelle. On comptait déjà parmi celle-ci, en 1991, " une proportion plus élevée de personnes touchant un revenu inférieur à 10000 \$ (17\%) ou compris entre 10000 et $19999 \$$ (15\%) »(CPSOC 1999a : 88). Et en 1996, on retrouve des indices marqués de pauvreté dans les quartiers où la population de francophones est élevée ou très élevée, soit à Vanier (51,9\%), Cumberland (35,2 \%) et Gloucester (27,4 \%) (CPSOC 1999a : 78). Ces secteurs comptent ainsi un pourcentage supérieur à la moyenne de familles monoparentales et de personnes vivant sous le seuil de pauvreté ou dont le revenu moyen est faible (CPSOC 1999a : 28). À Vanier, par exemple, le pourcentage de familles à faible revenu est de $34,5 \%$ et celui de personnes à faible revenu, de 50,1\% (CPSOC 1999a : 78-80). 
"...la proportion des aînées et aînés francophones vivant sous le seuil de faible revenu est plus élevée que dans l'ensemble de la population âgée de la province..."
C'est aussi dans ces quartiers que vit la plus forte concentration de personnes aînées francophones. À Vanier, $77 \%$ des personnes âgées sont francophones, 38, 5 \% de celles-ci le sont à Cumberland et $33 \%$ à Gloucester (CPSOC 1999a :28). Ces chiffres prennent une signification davantage importante si l'on tient compte du fait que la proportion des aînées et aînés francophones vivant sous le seuil de faible revenu est plus élevée que dans l'ensemble de la population âgée de la province (20, 5 \% comparativement à 16,8 $\%)$. Qui plus est, les femmes (25,8\%) sont deux fois plus susceptibles que les hommes francophones $(13 \%)$ de vivre sous le seuil de faible revenu (Office des affaires francophones 1999d :17).

\section{Une réalité autre que statistique}

Parler de pauvreté, c'est aussi prendre en considération son incidence dans la vie des individus et des familles. Ces dernières font face à des problèmes quotidiens pour se nourrir, se loger, se procurer des vêtements et des produits d'hygiène, payer le téléphone et l'électricité, se déplacer et chercher de l'emploi (Rapport du Comité organisateur 1998 : 52-54). Ainsi l'enquête de 1996 du Service de la santé de la Municipalité régionale d'Ottawa-Carleton (MROC) auprès des parents de la région démontre le sentiment d'insécurité qui les habite face à l'inquiétude de ne pas pouvoir offrir des soins adéquats à leur famille :

...25\% des parents d'enfants de 14 ans et moins s'inquiétaient "souvent " ou " très souvent " de leur capacité de payer les comptes. Par ailleurs $18 \%$ des familles qui gagnent moins de $30000 \$$ par année s'inquiètent de ne pas avoir assez d'argent pour se loger. Dix-neuf pour cent d'entre elles s'inquiètent de ne pouvoir acheter suffisamment de nourriture (CPSOC 1999a: 6).

D'autre part, entre 1991 et 1996, " on enregistrait une hausse de $32 \%$ du nombre de ménages risquant d'avoir des problèmes 
"Cette situation de pauvreté dans la région ...elle a été exacerbée par les réformes de l'aide sociale et par les coupures dans l'ensemble des services sociaux et de santé suite à l'élection du gouvernement conservateur en 1995. » d'accès à un logement abordable »(CPSOC 1999a : 5). Pour les personnes ayant témoigné aux Audiences populaires sur la pauvreté (Comité organisateur 1998), les problèmes liés au logement constituent la source de préoccupation le plus souvent évoquée après celle de l'insécurité alimentaire. L'entrée en vigueur de la Loi codifiant et révisant le droit de la location à usage d'habitation (loi 96) en 1998, qui abolit le contrôle des loyers lors de nouvelles locations, conjuguée aux coupures dans les prestations d'aide sociale et au faible taux de disponibilité de logements locatifs dans la région d'Ottawa (de l'ordre de 0,7 \% en 1999), complexifie davantage le problème.

Cette situation de pauvreté dans la région n'est pas nouvelle mais elle a été exacerbée par les réformes de l'aide sociale et par les coupures dans l'ensemble des services sociaux et de santé suite à l'élection du gouvernement conservateur en 1995. Ces coupures de l'ordre de 21,6\% dans les prestations d'assistance sociale, justifiées, selon ce gouvernement, par la nécessité de ramener les taux de l'Ontario à des niveaux semblables à ceux des autres provinces canadiennes (Moscovitch 1997 : 85), ont fait basculer les personnes déjà aux prises avec des difficultés financières importantes dans un état de survivance de plus en plus précaire. Des bénéficiaires de l'aide sociale soulignent concrètement leurs difficultés après les coupures :

Certains indiquaient qu'ils souffraient de faim, qu'ils se sentaient dégradés, qu'ils avaient peur pour leurs enfants et qu'ils se sentaient humiliés par l'attitude des travailleurs socianx an burean d'aide sociale. [...] Certains s'inquiétaient de la détérioration de leur état de santé (causé par le stress, l'anxiété etc.), par la crainte que des médicaments ne seraient pas couverts, par la dépression et par la malnutrition (Rapport de l'équipe $1999: 30)$.

Les médias et les discours politiques choisissent néanmoins la plupart du temps de faire abstraction de ces réalités en centrant plutôt les débats entourant la pauvreté sur la pertinence des seuils utilisés pour la mesurer. Ce faisant, ils contournent les vrais enjeux 
"Ces situations de précarité ont par ailleurs fait l'objet de nombreuses analyses qui font état, entre autres, des " processus de marginalisation "...auxquels sont exposées les personnes qui en sont marquées et de la "production sociale de la désinsertion" (de Gaulejac et Taboada ...qui mène à l'exclusion." et se cantonnent dans une interprétation quantitative de la pauvreté, vue uniquement comme une affaire soit de chiffres, soit de manques. Or, aborder la pauvreté uniquement sur la plan économique, c'est faire abstraction de la «mise à part [...] perçue non seulement comme une caractéristique de vie de celui qui n'a rien, mais comme sa pauvreté même" (Sassier 1990:350). L'isolement et la fragilisation des liens sociaux guettent constamment les personnes dont la vie est marquée par l'extrême précarité économique. Ils «affectent les gens à la fois dans leur équilibre personnel, leur image de soi et leurs relations à autrui " (Blais 1997 : 14), bref, dans toutes les dimensions de leur vie dont "la bouffe et les amours " (ibid). Ces situations de précarité ont par ailleurs fait l'objet de nombreuses analyses qui font état, entre autres, des "processus de marginalisation" (Castel 1994:11) auxquels sont exposées les personnes qui en sont marquées et de la " production sociale de la désinsertion » (de Gaulejac et Taboada Léonetti 1994 : 79) qui mène à l'exclusion.

Cette précarité des liens sociaux parsème d'ailleurs le discours des personnes ayant offert des témoignages dans le cadre des audiences populaires sur la pauvreté :

$$
\begin{aligned}
& \text { "... Être limité dans ses déplacements, c'est rétrécir } \\
& \text { l'éclairage social, le contact avec la vie active qui sont } \\
& \text { pourtant essentiels à la santé mentale "; "...il est } \\
& \text { essentiel d'assurer un appui à ceux dont la vie ressemble } \\
& \text { à un entonnoir et qui ont encore assez d'espoir pour } \\
& \text { ne pas couler" (Rapport du Comité organisateur } \\
& 1998 \text { : 105). }
\end{aligned}
$$

Les personnes qui participent à des initiatives pour contrer la pauvreté cherchent évidemment des moyens pour pallier l'instabilité économique dans laquelle les a plongées la perte d'un emploi, les coupures dans les programmes sociaux, mais elles cherchent aussi à tisser des liens, à développer des solidarités, à s'épauler pour affronter les vicissitudes de leur vécu individuel et collectif. La participation aux activités d'entraide contribue au développement « d'une identité d'acteur chez des gens que l'utilisation des services caritatifs ou de soutien public cantonnait dans un 
statut d'assistés » (Fréchette $2000: 10$ ). N'existant, pour la plupart, que depuis quelques années et constamment confrontées à de sérieuses contraintes, notamment financières, ces activités d'entraide ne réussissent cependant pas à pallier l'insuffisance économique vécue au quotidien par la plupart des personnes qui y participent. S'organisent donc, au cours des dernières années, côte à côte et souvent au sein des mêmes organismes communautaires, des services de type caritatif, tels des banques alimentaires, et des projets innovateurs axés sur des pratiques de solidarité et d'entraide.

\section{Les comptoirs alimentaires, une réponse à la faim}

Les comptoirs alimentaires ont une longue histoire dans la région d'Ottawa-Carleton. En effet, le dispensaire des Soeurs de la Charité, créé en 1845, peu après l'établissement de cette communauté religieuse à Bytown (nom d'Ottawa à l'époque), continue, depuis lors, à offrir du dépannage alimentaire. Par contre, la plupart des banques d'alimentation qui existent aujourd'hui ont été établies depuis les années 80. Dans les communautés anglophones, ces comptoirs sont souvent l'oeuvre d'une église ou d'un regroupement d'églises de même appartenance confessionnelle. Par contre, ce type d'initiative existe peu dans les paroisses francophones, du moins dans celles de la région d'Ottawa. Les comptoirs situés dans les quartiers où l'on retrouve les plus fortes concentrations de personnes francophones ont généralement été mis sur pied par ou avec l'appui et la collaboration des centres de ressources communautaires. À Cumberland et à Overbrook-Forbes, par exemple, les banques alimentaires font partie de l'ensemble de services offerts par les centres locaux de ressources, alors que dans le quartier de la Basse-Ville, un comptoir alimentaire a été créé récemment au sein de la Maison communautaire du bon voisinage $^{7}$. À Vanier et à Gloucester, par contre, les Centres de ressources ne sont pas dotés de comptoirs alimentaires, mais ils ont collaboré avec d'autres groupes et associations pour développer ces ressources dans leurs communautés respectives. Le comptoir 
"Les tensions soulevées par ces deux visions paradoxales ouvrent la voie à des réflexions sur d'autres formes de pratiques pour contrer la pauvreté." alimentaire de Gloucester, par exemple, qui existe depuis 1989, est un organisme de bienfaisance autonome qui maintient une étroite collaboration avec le centre communautaire de Gloucester alors que Partage Vanier, créé en 1991 avec la collaboration du centre communautaire de cette municipalité, demeure à ce jour une oeuvre de la congrégation des Soeurs de la Charité qui offre des services de dépannage à la communauté de Vanier.

Sans nier le rôle vital des banques alimentaires dans l'ensemble des stratégies auxquelles doivent avoir recours les familles et les individus pour combler leurs besoins essentiels, il importe néanmoins de dénoncer la nature assistancielle de ces pratiques. Elles perpétuent les représentations sociales des pauvres comme victimes qui « inspire[nt] de la pitié » et dont l'utilité sociale est de permettre "de se racheter une bonne conscience en faisant l'aumône (Ruffel-Chaffou 1996 : 387). Cette vision suscite une tension entre la représentation des personnes comme sujets plutôt que comme actrices sociales et les philosophies des centres de ressources communautaires qui veulent "promouvoir l'amélioration de la qualité de vie individuelle et collective " (Centre de ressources communautaires Overbrook-Forbes 1999 : 12) et développer des initiatives "en faveur du changement social " (Centre de ressources communautaires de Cumberland s.d.). Les tensions soulevées par ces deux visions paradoxales ouvrent la voie à des réflexions sur d'autres formes de pratiques pour contrer la pauvreté. C'est pourquoi, dès le début des années 90, incités par certaines réflexions déjà amorcées par la Coalition alimentaire d'Ottawa-Carleton (Ottawa-Carleton Coalition : 1992), les Centres de ressources communautaires et la MROC explorent des alternatives au problème de l'insécurité alimentaire.

\section{Les cuisines collectives, une alternative à la charité}

Le questionnement soulevé par le débat sur la portée sociale et symbolique des banques alimentaires vise un élargissement de la question de la faim comme problème individuel à celle plus globale 
de responsabilité collective et sociale face à la pauvreté. C'est de cette vision que s'inspirent les premières cuisines collectives francophones dans Ottawa-Carleton. Un premier projet pilote voit le jour dans la Basse-Ville à l'hiver 93, suivi l'année suivante, par la mise sur pied de quatre groupes de cuisines collectives communautaires dans le quartier de la Basse-Ville (CRBV :1994) et de deux autres groupes à Overbrook-Forbes (Centre de ressources communautaires Overbrook-Forbes : 1994) et à Cumberland (Centre de ressources de Cumberland : 1994). Ces deux derniers projets sont organisés et animés par le Service de gestion familiale de la MROC.

Dès leur implantation, on voit se profiler, au sein des premières cuisines, deux philosophies coexistantes. Au Centre de ressources de la Basse-Ville (CRBV), la cuisine privilégie une approche de développement communautaire, centrée sur le partage et sur l'échange entre les participantes. Dans une étude sur les cuisines collectives de l'époque, Manon Mongrain souligne la vision de la cuisine collective du CRBV, non pas comme une fin en soi, mais plutôt comme un "un lieu informel de prise de confiance, de pouvoir et d'expérimentation de rapports sociaux plus équitables, plus justes » (Mongrain 1997 : 90). Elle rapporte les propos des intervenantes selon qui les objectifs des cuisines se traduisent par "l'accroissement de l'estime personnel, de la prise de confiance et de la mise à profit des ressources environnantes " (1996:46).

"Au cours des années, les cuisines collectives se sont multipliées et transformées, propulsées par l'énergie des participantes qui $y$ trouvent une voix et un statut d'actrices sociales, pour se camper davantage " comme service de proximité lié au développement d'une communauté "..." S'inscrivant dans une autre vision, les cuisines collectives organisées dans le cadre du programme de gestion familiale de la MROC poursuivent un but plus éducatif, jumelant gestion du budget et saine alimentation.

Au cours des années, les cuisines collectives se sont multipliées et transformées, propulsées par l'énergie des participantes qui y trouvent une voix et un statut d'actrices sociales, pour se camper davantage "comme service de proximité lié au développement d'une communauté » (Fréchette $2000: 10$ ). Certaines participantes ont voulu mettre davantage l'accent sur le partage en y ajoutant, par exemple, des discussions thématiques, des ateliers de discussion sur l'estime de soi, alors que d'autres cuisines s'adressent davantage aux besoins exprimés par un groupe de femmes immigrantes. 
"L'activité générée par la participation dans les cuisines crée un terrain propice à l'éclosion d'un projet d'entraide communautaire qui voit le jour en $1995 \ldots$...
D'autres, par ailleurs, ont voulu investir le domaine économique. Par exemple, les participantes des cuisines collectives du CRBV envisagent la création d'un restaurant communautaire à l'image du Chic resto Pop à Montréal.

L'activité générée par la participation dans les cuisines crée un terrain propice à l'éclosion d'un projet d'entraide communautaire qui voit le jour en 1995, le fruit d'une concertation entre trois centres de ressources communautaires francophones (CSCV, CRBV, Overbrook-Forbes) et l'organisme Entraide budgétaire.

\section{Le projet d'entraide communautaire $\square$ : des pratiques mobilisatrices}

"S'ébauchant en fonction des idées proposées lors de ces réunions publiques, les activités générées prennent diverses formes et couleurs, qui sont l'expression de visions individuelles et collectives, de talents, d'habiletés et de stratégies de débrouillardise multiples et divers des participantes. "
L'objectif premier de ce projet d'entraide est de "permettre aux assistés sociaux d'identifier des solutions face à la pauvreté qui sévit actuellement dans la région et de publier massivement les solutions développées" (Entraide budgétaire 1995). Le projet obtient du financement dans le cadre du programme des subventions non renouvelables de la MROC et de Centraide, financement qui sera accordé pour les trois phases du projet s'échelonnant sur une période de trois ans.

Appuyée par un comité directeur composé de participantes et de participants, d'intervenants communautaires des Centres de ressource et de la directrice d'Entraide budgétaire, la coordonnatrice organise, au cours de la première année, une série de six rencontres publiques pour lancer le projet, recruter des membres et générer des suggestions d'activités ou d'initiatives. S'ébauchant en fonction des idées proposées lors de ces réunions publiques, les activités générées prennent diverses formes et couleurs, qui sont l'expression de visions individuelles et collectives, de talents, d'habiletés et de stratégies de débrouillardise multiples et divers des participantes. Les initiatives ainsi mises de l'avant s'inscrivent dans " une nouvelle modalité de conception des services sociaux à partir d'une impulsion réciprocitaire [...], exercée par un groupe 
de personnes pour elles-mêmes, qui prend en compte l'espace et le temps quotidien des individus pour lesquels elle est pratiquée; c'est pourquoi ces services peuvent être appelés services de proximité » (Laville 1993 : 146).

Dans une entrevue réalisée auprès des membres du Projet d'entraide et publiée dans Reflets, celles-ci donnent un aperçu des motivations et des attentes multiples qui suscitent leur participation :

"...moi, ce qui m'a amenée à participer au projet, c'est la recherche d'autonomie, de liberté personnelle, la possibilité de connaître d'autres ressources, de prendre des choses en main...de plus, au niveau économique, je savais que le projet m'aiderait à soulager les problèmes de ma famille ";

"...moi, je cherche à démolir les mythes reliés aux assistés sociaux";

"...je cherchais des solutions économiques viables permettant de survivre ";

"... pour briser l'isolement et trouver des solutions". (Malenfant : $1997: 3$ ).

"Les quatre nouvelles cuisines qui se créent se donnent des saveurs locales uniques, à l'image des visions des participantes...»
La première année du projet donne lieu à un foisonnement d'activités et d'initiatives d'entraide : la mise sur pied de quatre jardins communautaires (deux à Vanier, dont celui du Centre de ressources parents-enfants qui se définit davantage, selon les participantes, comme un jardin collectif, un à Overbrook et un dans la Basse-Ville); la formation de trois groupes d'achat de fruits et légumes frais en gros, connus sous le nom de Boîte Verte; l'organisation de diverses activités telles que la cueillette de fruits, la confection et la vente de conserves ainsi que des sorties autrement inaccessibles aux participants et à leurs enfants. De ce Projet d'entraide jaillit aussi un intérêt renouvelé pour les cuisines collectives. Les quatre nouvelles cuisines qui se créent se donnent des saveurs locales uniques, à l'image des visions des participantes : la cuisine du Centre francophone, développée avec la collaboration 
"...un groupe de

femmes immigrantes ayant créé une cuisine collective entreprend des démarches pour transformer celle-ci en coopérative de travail." de ce centre culturel et de loisirs implanté depuis de nombreuses années àVanier; la cuisine collective Méli-Mélo de la coopérative d'habitation Desloges située dans le quartier Overbrook-Forbes; une cuisine collective végétarienne et ethnoculturelle; et finalement, la cuisine collective multiculturelle « La Mang'Ail » àVanier qui, en plus de cuisiner, initie des levées de fonds et organise des ateliers pour explorer la possibilité de se transformer en coopérative de travail (Entraide budgétaire : 1996). Se dessine ainsi une variété d'initiatives visant à prime abord à pallier l'insuffisance financière par le biais de mécanismes répondant aux aspirations plus globales exprimées par les participantes, et qui se transforment rapidement en " moyens de développer des savoir-faire, un capital relationnel, des connaissances et des compétences qui augmentent les capacités d'agir et de se situer dans son environnement " (Roustang 2000 : XI).

L'évolution constatée dans les finalités et les modes d'organisation des cuisines collectives offre d'ailleurs une illustration convaincante de la capacité mobilisatrice des initiatives mises de l'avant dans le cadre de ce projet.Alors que la plupart des premières cuisines collectives de la région étaient initiées et organisées par des professionnelles dans le cadre de programmes de gestion familiale, celles créées suite à des rencontres publiques communautaires sont l'aboutissement et la concrétisation d'un processus de mobilisation et de prise en charge par des femmes qui choisissent ce moyen pour s'entraider et pour agir sur les conditions potentiellement démoralisantes associées à la pauvreté. Selon une classification des cuisines collectives en trois volets élaborée par Fréchette (2000 : 50-51), celles dont il est question ici se situent clairement dans la catégorie des cuisines "centrées sur une approche de développement communautaire ", axées sur "l'activation des solidarités interpersonnelles et de groupe [et sur des] stratégies d'organisation communautaire et de développement économique " (ibid), plutôt que dans celle des cuisines centrées sur la nutrition et sur l'éducation. À preuve, pendant la deuxième année du Projet d'entraide, au cours de laquelle se poursuivent plusieurs des initiatives amorcées l'année précédente, un groupe de femmes immigrantes ayant créé une cuisine collective entreprend des démarches pour transformer celle-ci en coopérative de 
travail. Leur incorporation concrétise la naissance du Centre d'intégration, de formation et de développement économique (CIFODE), une petite entreprise de femmes cuisinant des plats tropicaux, offrant un service de traiteur et ayant pignon sur rue avec l'ouverture d'un petit restaurant. Tout en diversifiant les sources d'appuis communautaires et en forgeant des alliances avec d'autres acteurs sociaux, notamment avec le personnel de l'hôtel de ville de Vanier, des politiciens municipaux et plusieurs commerçants de la région, les participantes continuent, au cours de la deuxième année du projet, à développer de nouvelles initiatives et à explorer des avenues possibles pour des cuisines, entre autres à OverbrookForbes. On voit poindre un intérêt grandissant pour des services de proximité diversifiés qui «se fondent sur [...] les relations et échanges symboliques qui tissent la trame journalière de la vie locale, les aspirations, les valeurs et les désirs des gens qui en sont les usagers " (Laville 1993 : 147). S'organisent ainsi à Vanier, à l'automne 1997, grâce au travail d'un groupe de mères, la Coopérative de gardiennage Les petits soleils, et dans la BasseVille, en décembre 1996, un magasin partage, fruit d'une collaboration entre le Patro d'Ottawa, le CRBV et la Maison communautaire du bon voisinage. Soixante-deux familles participent à cette activité et l'intérêt généré depuis lors en fait un événement annuel.

"La concrétisation

d'initiatives communautaires créées et gérées par des personnes, la plupart sans emploi ou assistées sociales,... marque une étape importante dans une démarche vers des approches basées sur une véritable collaboration et sur des actions concertées..."
La concrétisation d'initiatives communautaires créées et gérées par des personnes, la plupart sans emploi ou assistées sociales, conjuguée à l'objectif qu'elles se fixent de médiatiser leurs solutions pour contrer l'insuffisance de revenu, marque une étape importante dans une démarche vers des approches basées sur une véritable collaboration et sur des actions concertées (Join-Lambert : 1996) qui se situent au-delà de vagues efforts d'appropriation ou d'empowerment. Leur implication dans les pratiques émancipatoires renforce chez les participantes le désir de contribuer à l'éclatement de mythes et de préjugés omniprésents au sujet de l'assistance sociale. Alors que certaines participent à des entrevues avec les médias, d'autres contribuent à la création et à la publication d'un livret intitulé Histoires drôles et moins drôles sur l'assistance sociale (Projet d'entraide communautaire :s.d.) qui tente de remettre les pendules à l'heure face à certaines réalités de l'assistance sociale. 
Baptisée "Pizza et politique ", la dernière phase du projet d'entraide témoigne du pouvoir qui « apparaît parmi une pluralité d'individus, du fait qu'ils sont capables d'user de leur liberté pour agir de "concert" " (Join-Lambert 1996 : 228). Ce pouvoir se manifeste à la fois individuellement et collectivement alors que plusieurs participants choisissent de s'investir dans une nouvelle initiative qui voit le jour dans la région, les audiences populaires sur la pauvreté.

\section{Audiences populaires sur la pauvreté $\square$ : une voix citoyenne et collective}

\section{"Les Audiences populaires sur la} pauvreté se veulent un forum communautaire pour " donner aux gens accès à un environnent agréable et sûr où ils pourraient exprimer leurs difficultés et faire valoir leurs forces "..."
S'inspirant d'un modèle de mobilisation communautaire à Toronto et dans d'autres villes ontariennes, en 1997, un regroupement d'organismes, formé notamment du Conseil de planification sociale d'Ottawa-Carleton, de la Coalition des centres de ressources communautaires et d'Entraide budgétaire, se mobilise pour organiser des audiences sur la pauvreté au sein de différentes communautés de la région. Les Audiences populaires sur la pauvreté se veulent un forum communautaire pour « donner aux gens accès à un environnent agréable et sûr où ils pourraient exprimer leurs difficultés et faire valoir leurs forces " (Comité organisateur 1998 : 43). Cette démarche a pour but de créer un espace public au sein duquel des personnes habituellement laissées pour compte puissent prendre la parole, s'exprimer et prendre leur place en tant qu'actrices sociales.

Dans le cadre d'assemblées publiques organisées à divers endroits dans la région au cours du mois d'octobre 1997, les gens sont invités à présenter des témoignages portant essentiellement sur les réalités quotidiennes et les défis auxquels sont confrontées les personnes pauvres et leurs familles. Le comité organisateur suggère quelques questions pour faciliter les témoignages: "Qu'est-ce que les gouvernements et les communautés devraient savoir au sujet de l'effet des coupures dans les programmes sociaux 
sur nous et notre famille? » et « Qu'est-ce que nous, les personnes les plus directement touchées par les coupures, faisons pour améliorer notre sort? » (Comité organisateur 1998 :51). Le forum à l'Hôtel de ville de Vanier se déroule uniquement en français (d'autres forums sont bilingues ou anglophones) et plusieurs des personnes participant aux rencontres "Pizza et politique » du projet d'entraide y témoignent, soit en petits groupes, soit à titre individuel. Se racontant au moyen de récits, de poèmes et de courtes pièces de théâtre, les participantes et les participants relatent les circonstances les ayant menés à la pauvreté, les souffrances, les contraintes quotidiennes, les stratégies de débrouillardise et les moyens utilisés pour s'en sortir.

"La pauvreté c'est pas quelque chose que l'on choisit, c'est quelque chose dans lequel on tombe. J'ai déjà vécu avec un revenu de 50,000 \$, plus bonus. Aujourd'hui, après avoir été malade, je vis dans la pauvreté. Pour m'en sortir, j'ai d'abord cherché un endroit où je pouvais payer le loyer. Au bout de trois ans, j'ai trouvé un logement subventionné. J'ai participé à un jardin communautaire. J'ai participé à une cuisine collective. Parfois, je panique quand je n'ai pas été capable de payer mon Hydro..." (Comité organisateur 1998 : 99).

"Je cherche des partenaires pour écrire un livre sur les solutions à la pauvreté, sur les trucs que j'ai découverts de peine et de misère, sur les ressources qui existent dans la communauté mais qui sont cachées " (ibid).

"..." ensemble on se bat et on essaie de s'en sortir "; " on partage beaucoup et cela me donne du courage "..."
Des femmes se regroupent en table ronde pour offrir un témoignage axé sur leur implication dans les projets d'entraide : "au niveau financier, je me sens plus sécure, capable de faire des choses "; " ensemble on se bat et on essaie de s'en sortir "; " on partage beaucoup et cela me donne du courage » (102-103). Deux autres groupes présentent des pièces de théâtre, l'une intitulée Comment arrondir les fins de mois en créant une cuisine coopérative et 
"...cette approche met d'abord l'accent sur " la mobilisation des populations et quartiers défavorisés afin de participer au processus d'administration qui reconnaît leurs idées, leurs forces et compétences, leurs talents uniques et leur créativité »..." l'autre, La cuisine franco.

Suite à la présentation au Conseil de la MROC du compte rendu des audiences, celui-ci met sur pied un Groupe de travail sur la pauvreté, composé de représentants élus et délégués par les collectivités démunies et de délégués du gouvernement régional. Présidé conjointement par un représentant de ces deux entités, le Groupe de travail régional compte sur l'apport de groupes de concertation organisés géographiquement, qui se rencontrent régulièrement pour discuter d'enjeux portant sur différents thèmes soulevés lors des audiences populaires. C'est par le biais de ce mécanisme de concertation que les personnes francophones impliquées dans l'initiative "Pizza et politique " contribuent à l'élaboration de la série de recommandations formulées par le Groupe de travail et présentées au Conseil régional en février 2000, dans un rapport final intitulé Les gens d'abord : lever les obstacles par l'action (Groupe de travail sur la pauvreté : 2000). Les recommandations portent sur les thèmes suivants : le logement, les services essentiels, l'éducation, les services récréatifs, la sécurité alimentaire, les services de garde, le transport et l'emploi.

Le processus ayant conduit à la préparation du rapport final s'inspire de l'approche communautaire à la réduction de la pauvreté préconisée par l'Institut Caledon de politiques sociales et des principes qui la sous-tendent. Ceux-ci visent à "répondre aux besoins fondamentaux, lever les obstacles, bâtir les compétences et promouvoir le développement économique » (Groupe de travail 1999 : 10). Tout en prônant « la mobilisation de tous les secteurs impliqués afin de créer des occasions économiques et sociales pour les ménages à faible revenu » (Groupe de travail 1999:10), cette approche met d'abord l'accent sur «la mobilisation des populations et quartiers défavorisés afin de participer au processus d'administration qui reconnaît leurs idées, leurs forces et compétences, leurs talents uniques et leur créativité » (ibid). Pour assurer l'implication soutenue des personnes et des collectivités à faible revenu dans l'articulation des enjeux et dans l'élaboration des politiques les concernant, le Groupe de travail suggère que soient maintenus les mécanismes de consultation populaire auprès de groupes de concertation comme entre autres celui connu sous 
"Le risque ici est évidemment que le mouvement de participation populaire amorcé par les audiences sur la pauvreté perde de son autonomie et que sa capacité d'action soit récupérée par les intérêts politiques..." l'appellation de "Pizza et politique ». Dans cette optique, le Département des services sociaux de la MROC attribue, en juillet 2000 , une subvention à un organisme de la communauté pour l'embauche d'une personne qui assurera la coordination de la participation à titre de citoyennes et de citoyens des personnes généralement exclues et vivant dans la pauvreté. Le risque ici est évidemment que le mouvement de participation populaire amorcé par les audiences sur la pauvreté perde de son autonomie et que sa capacité d'action soit récupérée par les intérêts politiques des élus municipaux, d'autant plus que ces derniers doivent composer avec des contraintes et des marges de manoeuvre de plus en plus étroites imposées par les politiques du gouvernement conservateur en matière de services sociaux. Les liens sociaux générés par le mouvement des audiences populaires risqueraient alors de se transformer, devenant le "résultat " plutôt que "la condition " (Autès 1999 :216) de la politique, obnubilant ainsi la participation citoyenne, toujours fragile, des personnes vivant dans la précarité.

\section{Conclusion}

"...axés sur la solidarité, les services de proximité qui se développent "ne trouvent plus origine dans les déficiences présentées par certains publics cibles et identifiés par rapport à une supposée normalité..."
Les initiatives contre la pauvreté dont il est ici question signalent des changements importants dans les façons d'aborder la pauvreté et de travailler avec les personnes démunies dans les milieux francophones de l'Ontario, changements qui se profilent sur deux plans convergeant vers des pratiques émancipatoires. On constate d'abord l'émergence de pratiques qui marquent une rupture dans les modalités assistancielles établies principalement par les communautés religieuses et jusqu'alors fortement ancrées dans une vision de charité ne s'adressant qu'aux pauvres méritants (Sassier 1990). Par ailleurs, axés sur la solidarité, les services de proximité qui se développent " ne trouvent plus origine dans les déficiences présentées par certains publics cibles et identifiés par rapport à une supposée normalité mais dans l'interrogation collective sur le vécu quotidien des intéressés " (Laville 1993 : 147). S'amorce alors l'esquisse d'un deuxième changement dans 
"Ce travail ne peut continuer à se faire que dans la mesure où les actrices et les acteurs sociaux impliqués feront preuve de vision et de créativité pour continuer à développer des approches communautaires ancrées dans des pratiques émancipatoires et inclusives. » les pratiques de lutte contre la pauvreté, celui-ci émanant de la création d'un contexte de travail communautaire avec les pauvres plutôt que pour les pauvres. Ce travail ne peut continuer à se faire que dans la mesure où les actrices et les acteurs sociaux impliqués feront preuve de vision et de créativité pour continuer à développer des approches communautaires ancrées dans des pratiques émancipatoires et inclusives. En ouvrant la voie à la création de lieux d'expression pour un groupe de personnes jusqu'alors exclues, ces pratiques doivent par ailleurs interpeller l'ensemble de la communauté franco-ontarienne parce qu'elles contribuent à forger une vision de la citoyenneté qui dépasse la connotation souvent étroite de celle-ci pour devenir non plus « un état, mais une action " (Hamel 2000 : 66). Cette vision de la citoyenneté, qui consiste "à prendre pied dans la société en faisant corps avec ses semblables pour défendre son existence, assurer sa survie " (ibid), peut contribuer au développement économique et social de la communauté franco-ontarienne dans la mesure où les pratiques qui s'y créent participent à l'émancipation de toutes et de tous.

\section{Bibliographie}

ANDREW, Caroline, Lyne BOUCHARD, Francoise BOUDREAU, Linda CARDINAL, Diane FARMER, Michèle KÉRISIT, Denise LEMIRE (1997). Conditions de possibilité des services de santé et des services sociaux en français en Ontario : un enjeu pour les femmes, Ottawa, Table féministe francophone de concertation provinciale de l'Ontario.

ASSOCIATION CANADIENNE-FRANÇAISE DE L'ONTARIO(1985). Les francophones tels qu'ils sont. Regard sur le monde du travail franco-ontarien, Ottawa, ACFO.

AUTÈS, Michel (1999). Les paradoxes du travail social, Paris, Dunod.

BAGAOUI, Rachid et Donald DENNIE (1999). «Le développement économique communautaire : nouveau départ pour le mouvement associatif franco-ontarien ", Reflets, Revue ontaroise d'intervention sociale et communautaire, $5,1: 55-94$.

BLAIS, Louise (1997). «La question de la santé mentale en milieu de pauvreté : masque ou vitrine du pouvoir et de l'oppression ", Revue canadienne de santé mentale communautaire, 16,1 :5-22.

BRUN, Patrick (1996). "Pour une connaissance émancipatoire des plus pauvres : Jürgen Habermas et Joseph Wresinski ", dans Fontaine, Pierre (dir.), La connaissance des pauvres, Louvain-la-Neuve, 
Les Éditions Travailler le Social.

BUREAU, Brigitte (1989). Mêlez-vous de vos affaires. Vingt ans de luttes franco-ontariennes, Vanier, Association canadienne-française de l'Ontario.

CARDINAL, Linda (1998). "Des femmes d'action :l'autre histoire de l'Ontario français de 1969 à 1982 ", dans Hébert, Monique, Nathalie Kermoal et Phillis Leblanc (dir.), Entre le quotidien et le politique : Facettes de l'histoire des femmes francophones en milieu minoritaire, Ottawa, RNAÉF, 159193.

CARDINAL, Linda et Cécile CODERRE (1990). "Les francophones telles qu'elles sont : les Ontaroises et l'économie ", Revue du Nouvel-Ontario, no 12,151-181.

CARRIÈRE, Fernan (1993). "La métamorphose de la communauté franco-ontarienne, 1960-1985 ", Cornelius J.Jaenen (s. la dir. de), Les Franco-Ontariens, Ottawa, Les Presses de l'Université d'Ottawa, 304-340.

CASTEL, Robert (1994). «La dynamique des processus de marginalisation : de la vulnérabilité à la désaffiliation ", Cahiers de recherche sociologique, no 22.

CENTRE DE RESSOUR CES COMMUNAUTAIRES DE LA BASSE-VILLE(1993). Programme de développement communautaire. Activités 1993-1994, document mineo.

CENTRE DE RESSOURCES COMMUNAUTAIRES D'OVERBROOK-FORBES (1994). Rapport du Directeur général, document mineo.

CHOLETTE, Chantal (1998). « Le travail obligatoire en Ontario : solution ou imposture? », Reflets, Revue ontaroise d'intervention sociale et communautaire, $4: 1,100-126$.

COMITÉ OR GANISATEUR (1998). «Les gens d'abord » Audiences populaires sur la pauvreté, Ottawa, Rapport du Comité organisateur.

CONSEIL CANADIEN DE DÉVELOPPEMENT SOCIAL (1999). Le bien-être de l'enfant et le revenu familial, un nouveau regard au débat sur la pauvreté, Ottawa, CCDS.

CONSEIL DE PLANIFICATION SOCIALE D'OTTAWA-CARLETON et CENTRAIDE

D'OTTAWA-CARLETON (1999a). Une histoire de deux mondes. Tendances économiques et sociodémographiques à Ottawa-Carleton. Rapport technique. Ottawa, CPSOC-Centraide OC.

CONSEIL DE PLANIFICATION SOCIALE D'OTTAWA-CARLETON et CENTRAIDE D'OTTAWA-CARLETON (1999b). Une histoire de deux mondes. Tendances économiques et sociodémographiques à Ottawa-Carleton. Ottawa, CPSOC-Centraide OC.

CONSEIL DE PLANIFICATION SOCIALE D'OTTAWA-CARLETON (2000). Le marché de l'emploi est-il travaillable? Ottawa, Conseil de planification sociale.

DALPÉ, Jean-Marc (1980). Les murs de nos villages, Sudbury, Prise de Parole.

de GAULEJAC,Vincent et Isabel TABOADA LÉONETTI (1994). La lutte des places, Paris, Desclée de Brouwer.

DIALLO, Lamine et REITSMA-STREET, Marge (1995). "Stratégies de survie et d'identité : les dynamiques culturelles dans un projet d'intervention en prévention communautaire ", Reflets, Revue ontaroise d'intervention sociale et communautaire, 1 : 1, 43-69.

FARMER, Diane et Jeff POIRIER (1999). "La société et les réalités francophones en Ontario », dans JosephYvon Thériault (dir.), Francophonies minoritaires au Canada, Moncton, Éditions d'Acadie, 265-281. 
FÉDÉRATION DES FEMMES CANADIENNES-FRANÇAISES (1983). Seules en grand nombre, Dossier socio-économique sur la situation des Ontaroises chefs de famille monoparentale, Ottawa, FFCF. FÉDÉRATION NATIONALE DES FEMMES CANADIENNES-FRANÇAISES (1986). Le réflexe du meilleur. Trousse d'information à l'intention des cheffes de famille monoparentale ontaroises, Ottawa, FNFCF.

FRÉCHETTE, Lucie (2000). Entraide et services de proximité. L'expérience des cuisines collectives, SainteFoy, Presses de l'Université du Québec.

GILBERT, Anne (1999). Espaces franco-ontariens, Ottawa, Le Nordir.

GROUPE DE TRAVAIL SUR LA PAUVRETÉ (1999). Les gens d'abord : créer de l'espoir par le changement, Rapport provisoire du Groupe de travail sur la pauvreté dans la région d'OttawaCarleton.

GROUPE DETRAVAIL SUR LA PAUVRETÉ (2000). Les gens d'abord :lever les obstacles parl'action, Ottawa-Carleton, Rapport final du Groupe de travail sur la pauvreté.

GROUPE D'ÉTUDE POUR L'EMPLOI (1999). Partenaires pour l'emploi Investir dans Ottawa-Carleton, Ottawa, Partenaires pour l'emploi d'Ottawa-Carleton.

HAMEL, Jacques (2000). "Citoyenneté, jeunes et droits sociaux », dans Boisvert,Yves, Jacques Hamel et Marc Molgat (dir.), Vivre la citoyenneté Identité, appartenance et participation, Montréal, Liber.

HUNTLEY, Sandra (1998). La main d'oeuvre invisible de la région d'Ottawa-Carleton, Ottawa, Société d'expansion économique d'Ottawa.

INSTITUT CANADIEN D'ÉDUCATION DES ADULTES (ICÉA) avec la collaboration de la Fédération des francophones hors Québec (FFHQ) (1989). En toutes lettres et en français. L'analphabétisme et l'alphabétisation des francophones au Canada, Montréal, ICÉA.

JUTEAU, Danielle et Lise SÉGUIN-KIMPTON (1993). "La collectivité franco-ontarienne : structuration d'un espace symbolique et politique ", dans Cornelius J. Jaenen (dir.), Le FrancoOntariens, Ottawa, Les Presses de l'Université d'Ottawa, 265-304.

LAMOUREUX, Jocelyne (2000). "Représentations et pratiques citoyennes dans le mouvement communautaire autonome ", dans Yves Boisvert, Jacques Hamel et Marc Molgat (dir.), Vivre la citoyenneté. Identité, appartenance et participation, Montréal, Liber.

LAVILLE, Jean-Louis (1993). Les services de proximité en Europe, Paris, Syros.

MALENFANT, Brigitte (1997). Réflexions sur un projet d'entraide communautaire, Reflets, Revue ontaroise d'intervention sociale et communautaire, vol.3, no.1, 16-28.

MICHAUD, Jacinthe (1999). «Les femmes francophones et le travail obligatoire : un enjeu pour l'économie sociale ", Reflets, Revue ontaroise d'intervention sociale et communautaire, 5,1:95- 113.

MONGRAIN, Manon (1997). "Cuisines collectives : modèle alternatif d'organisation sociale et économique ", Reflets, Revue ontaroise d'intervention sociale et communautaire, $3: 1,80-100$.

MONGRAIN, Manon (1996). Des fourmis qu'on méprend pour des cigales : les cuisines collectives comme modèle alternatif d'organisation sociale et économique, mémoire de maîtrise, École de service social, Université d'Ottawa.

MUNICIPALITÉ RÉGIONALE D'OTTAWA-CARLETON (1996). Rapport sur l'état de santé des enfants et des familles, Ottawa, Service de la santé.

OFFICE DES AFFAIRES FRANCOPHONES (1999a). Les francophones en Ontario. Profil statistique. Toronto, OAF. 
OFFICE DES AFFAIRES FRANCOPHONES (1999b). Les femmes francophones en Ontario. Profil statistique. Toronto, OAF.

OFFICE DES AFFAIRES FRANCOPHONES (1999c). Les jeunes francophones en Ontario. Profil statistique. Toronto, OAF.

OFFICE DES AFFAIRES FRANCOPHONES (1999d). Les personnes âgées francophones en Ontario. Profil statistique. Toronto, OAF.

OTTAWA-CARLETON COALITION (1992). Overdrawn at the Food Banks :The Hunger Crisis in Ottawa-Carleton, The Ottawa-Carleton Coalition Project.

PARÉ, François (1994). Les littératures de l'exiguité, Ottawa, Le Nordir.

RAPPORT DE L'ÉQUIPE DE PROJET SUR LE SUIVI DU PROGRAMME ONTARIO AU TRAVAIL (1999). Dire les choses telles qu'elles sont : espoir et réalité. Expériences de participantes et de participants au programme Ontario au travail, Ottawa, MROC.

RAPPORT DU COMITÉ ORGANISATEUR (1998). "People First-Les gens d'abord » Audiences populaires sur la pauvreté, Ottawa, MROC.

PROJET D'ENTRAIDE COMMUNAUTAIRE (s.d.). Histoires drôles et moins drôles sur l'assistance sociale, Vanier, Entraide budgétaire.

RUFFEL-CHATTOU, Pascale (1996). «À quoi joue-t-on quand on s'implique dans une recherche sur les pauvres? ", dans Fontaine, Pierre (dir.), La connaissance des pauvres, Louvain-la-Neuve, Les Éditions Travailler le Social

SASSIER, Philippe (1990). Du bon usage des pauvres : histoire d'un thème politique XV1e - XXe siècle, Paris, Fayard.

TAHON, Marie-Blanche (1995). La famille désinstituée. Introduction à la sociologie de la famille, Ottawa, Les Presses de l'Université d'Ottawa.

WAGNER, Serge (1991). Analphabétisme de minorité et alphabétisation d'affirmation nationale à propos de l'Ontario français, Toronto, Gouvernement de l'Ontario.

WELCH, David (1999). "L'économie sociale en Ontario français : analyse historique, pratiques actuelles et recherche de sens ", Reflets, Revue ontaroise d'intervention sociale et communautaire, 5,1: 54-74.

WELCH, David (1995). "Les Franco-Ontariens : la résistance comme mode de vie », Reflets, Revue ontaroise d'intervention sociale et communautaire, 1-1:20-42.

\section{Rapports d'organismes et fonds d'archives dépouillés}

Centre de ressources de la Basse-Ville. Rapports annuels pour les années 1989-90 à 1998-99.

Centre de ressources communautaires de Cumberland. Rapports annuels de 1989-90 à 1998-99.

Centre de ressources communautaires de Gluocester. Rapports annuels de 1989-90 à 1999.

Centre de ressources communautaires Overbrook-Forbes. Rapports annuels pour les années 1990-91 à 1999.

Centre des services communautaires de Vanier. Rapports annuels pour les années 1989-90 à 1998-99. Entraide budgétaire. Demandes de subvention aux Services sociaux de la MROC 1995, 1996 et 1997. 
Entraide budgétaire. Projet d'entraide : Rapports d'activités et d'évaluation des phases I (1996-97); Rapport d'activités et évaluation de la phase II (1997-98); Rapports mensuels des activités de la phase III (1998-99).

Fonds du Comité de Réveil de la Basse-Ville. Fonds du Centre de recherche en civilisation canadiennefrançaise. (C57), Université d'Ottawa, CRCCF.

\section{Notes}

1. Les auteures désirent remercier Sophie Bergeron et Magnolia Soutyrine, étudiantes à l'École de service social de l'Université d'Ottawa, qui ont participé à la recherche des documents d'archives dont il est question dans cet article.

2. Dans le portrait le plus récent présenté par les Affaires francophones, on pourrait croire que les francophones sont favorisés et ce à divers niveaux : poursuite des études universitaires, revenus plus élevés que la population en général et même écarts moins élevés entre les revenus d'emploi moyens des femmes et des hommes (Office des affaires francophones 1999a :1) Ce portrait optimiste est toutefois mis en veilleuse dans une analyse plus fine des données du moins en termes de scolarité. En effet, l'Office souligne que «le niveau de scolarité des francophones tend à être inférieur à celui de la population en général » (Office des affaires francophones 2000a : 11).

3. Quoiqu'aucun des treize centres de ressources communautaires de la région d'Ottawa-Carleton n'offre des services exclusivement en français, cinq de ceux-ci assurent les services à un bassin important de personnes francophones. Il s'agit des Centres de ressources communautaires de la Basse-Ville, de Gloucester et de Cumberland, de l'Association des services communautaires d'Overbrook-Forbes et du Centre de services communautaires de Vanier.

4. Les activités francophones en matière d'alphabétisation se sont organisées principalement à la base. Elles sont le fait de groupes communautaires convaincus que l'analphabétisme est un problème collectif et qu'il faut s'alphabétiser ensemble. Il y a eu certes des projets-pilotes dans les collèges, mais le mouvement est d'abord et avant tout marqué par des initiatives communautaires-populaires, qui depuis l'Est ontarien se sont diffusées à l'échelle de la province (Gilbert 1999 : 66-67).

5. Les seuils de pauvreté et de faible revenu sont deux indices économiques qui permettent de mesurer les inégalités économiques touchant les individus et les familles. Le Conseil canadien de développement social définit le seuil de pauvreté comme un niveau inacceptable d'inégalité des revenus alors que Statistique Canada a établi les seuils de faible revenu selon le revenu considéré suffisant pour que les familles ne vivent pas dans la gêne (CCDS1999:36). Les seuils de faible revenu (SFR) établissent 35 différents seuils de revenu pour les familles économiques et les personnes seules, selon la taille de la famille et celle de la collectivité. Les familles et les personnes seules dont les dépenses consacrées à la nourriture, aux vêtements et au logement sont de plus de $20 \%$ supérieures à la moyenne sont considérées comme étant dans la gêne (CPSOC 1999b :14).

6. Le terme famille gynéparentale utilisé par Marie-BlancheTahon (1995) tient compte de la responsabilité des femmes comme parente de même que du choix des femmes d'assumer ce rôle de mère.

7. Situées dans plusieurs quartiers où se retrouve une concentration importante de logements sociaux, les maisons communautaires sont généralement mises sur pied et gérées par des associations de résidants et de résidantes de ces quartiers et occupent une unité prêtée par la corporation de logement social. Ces maisons communautaires travaillent par ailleurs en étroite 